\title{
Clinical and electrocardiographic characteristics at admission of COVID-19/SARS-CoV2 pneumonia infection
}

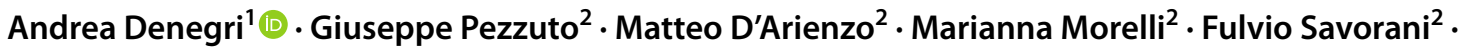 \\ Carlo G. Cappello ${ }^{1} \cdot$ Antonio Luciani $^{2} \cdot$ Giuseppe Boriani ${ }^{1}$ (I)
}

Received: 25 July 2020 / Accepted: 17 November 2020 / Published online: 4 January 2021

(c) Società Italiana di Medicina Interna (SIMI) 2021

\begin{abstract}
Background The aim of the present study was to compare clinical and electrocardiographic characteristics of patients with COVID-19 pneumonia in Modena, Emilia Romagna, Italy.

Methods Patients admitted to the emergency department for suspected COVID-19 pneumonia from March the 16th to April the 15th were enrolled in the study. COVID-19 pneumonia was confirmed by positive nasopharyngeal swab. Primary endpoint was 30-day mortality.

Results 201 patients were diagnosed with COVID-19 pneumonia. Compared to survivors, patients who died were older $(79.7 \pm 10.8$ vs $65.6 \pm 14.1, p<0.001)$, with a more complex cardiovascular history, including coronary artery disease (CAD, $33.3 \%$ vs $13.3 \%, p=0.004)$, atrial fibrillation $(23.8$ vs $8.8, p=0.011)$ and chronic kidney disease (CKD $35.7 \%$ vs $7.0 \%$, $p<0.001$ ). 30-day mortality was $20,9 \%$ in these patients; atrial fibrillation (OR $12.74,95 \%$ CI $3.65-44.48, p<0.001$ ), STsegment depression (OR 5.30, 95\% CI 1.50-18.81, $p=0.010$ ) and QTc-interval prolongation (OR 3.17, 95\% CI 1.24-8.10, $p=0.016)$ at ECG admission were associated to an increased mortality risk. On the contrary, sinus rhythm (OR $0.08,95 \% \mathrm{CI}$ $0.02-0.27, p<0.001)$ and low-molecular weight heparin (LMWH) administration (OR 0.08, 95\% CI 0.02-0.29, $p<0.001$ ) were related to reduced mortality. At multivariate analysis, after adjustment for age, sex, diabetes, CAD, and MCA admission, sinus rhythm (HR 2.7, CI 95\% 1.1-7.0, $p=0.038$ ) and LMWH (HR 8.5, 95\% CI 2.0-36.6, $p=0.004$ ) were confirmed to be independent predictors of increased survival.
\end{abstract}

Conclusion Sinus rhythm at ECG admission in COVID-19 pneumonia patients was associated with greater survival as well as LMWH administration, which conferred an overall better outcome.

Keywords COVD19 $\cdot$ SARS/CoV2 $\cdot$ ECG $\cdot$ Cardiovascular disease $\cdot$ Mortality

\section{Introduction}

Coronavirus disease (COVID-19) with Severe Acute Respiratory Syndrome (SARS) due to a novel Coronavirus (CoV-2) has rapidly spread worldwide; as of today September the 20th, World Health Organization has declared 30.6 millions confirmed cases of COVID-19, including 950.000 deaths

Andrea Denegri

denegri.andrea@aou.mo.it

1 Cardiology Division, Department of Biomedical, Metabolic and Neural Sciences, University of Modena and Reggio Emilia, Azienda Ospedaliero-Universitaria Di Modena, Largo del Pozzo, 71, 41125 Modena, Italy

2 Emergency Department, Azienda Ospedaliero-Universitaria Di Modena, Largo del Pozzo, 71, 41125 Modena, Italy
[1]. COVID-19 pandemic outbroke in our regions at the end of Februry, and the majority of cases were registered in northern Italy, with a significant impact on cardiovascular hospitalization rate [2]. Clinical manifestations of infection are characterized by fever, cough and dyspnea, with severe case of infection and intensive care unit (ICU) admission up to $15 \%$ and $5 \%$ of case, respectively [3]. It is well known that COVID-19 may affect the cardiovascular system and several studies have described myocarditis, acute coronary syndrome or heart failure as clinical manifestation of the disease [4]. In this regard, electrocardiography (ECG) may be useful to discern acute myocardial injury or rhythm alterations in COVID-19 patients. ECG characteristics in COVID-19 pneumonia have been already described in previous studies, but their relations to outcomes are still debated [5-7]. The aim of the present study was, indeed, to explore 
ECG abnormalities at admission in all-comer COVID-19 patients admitted to the Emergency Department of Modena University Hospital.

\section{Methods}

All-comer patients admitted to the Emergency Department of Modena University Hospital with suspected COVID-19 pneumonia from March the 16th to April the 15th 2020 were enrolled in the study. All patients with typical respiratory symptoms have been diagnosed with COVID-19 infection by positive nasopharyngeal swab and typical radiological features at chest X-ray. Clinical data, including outcome, were extracted from medical records. Venous and arterial blood samples for blood gas analysis were collected at the time of hospitalization and there after based on clinical indication. 12-lead ECG with $25 \mathrm{~mm} / \mathrm{s}$ and $1 \mathrm{mV} / \mathrm{cm}$ calibration and $0.05-150 \mathrm{~Hz}$ filter setting. ECG traces were recorded and analyzed off-line. The following ECG parameters were considered: heart rate (HR), presence of ST-T abnormalities and corrected QT interval (ms), this latter measured as the time between the start of $\mathrm{Q}$ wave and the end of the $\mathrm{T}$ wave, corrected by HR according to Bazett's formula. Rhythm and conduction alterations were also considered.

\section{Statistical analysis}

Continuous variables were expressed as mean \pm standard deviation (SD), while categorical data were expressed as absolute values and proportions. Variables were compared between patient survivors and non-survivors at 30 day using the Fisher exact test for categorical variables, and the $t$ test or the Mann-Whitney $U$ test, as appropriate, for continuous variables. Survival curves were plotted using the Kaplan-Meier method with log-rank test and COXregression model was applied. Analyses were performed with SPSS, Statistical Package for Social Science) software (v26, SPSS Inc., Chicago, IL, USA). For all the statistical analyses, $p<0.05$ was considered significant.

\section{Results}

Patients baseline characteristics are resumed in Table 1. Mean age was $68.5 \pm 14.7$ years; women represented $35.8 \%$ of the study population. Fever $(92.5 \%)$ was the most prevalent symptom at admission; dyspnea $(68.7 \%)$, cough (49.8\%) and gastrointestinal symptoms (19.4\%) were also detected although in lower percentage. Only six patients have complained chest pain. Vital parameter measurement at admission revealed a systolic blood pressure of $132.5 \pm 21.9 \mathrm{mmHg}$, heart rate (HR) $91.1 \pm 17.0 \mathrm{bpm}$,
Table 1 Baseline characteristics

\begin{tabular}{lllr}
\hline & Dead $(n=42)$ & Alive $(n=159)$ & \multicolumn{1}{l}{$p$} \\
\hline Age & $79.7 \pm 10.8$ & $65.6 \pm 14.1$ & $<0.001$ \\
Female sex & 40.5 & 34.6 & 0.297 \\
Hypertension & 64.3 & 54.4 & 0.166 \\
Diabetes & 26.2 & 16.5 & 0.113 \\
Dyslipidemia & 16.7 & 18.4 & 0.501 \\
COPD & 4.9 & 7.0 & 0.475 \\
Smoking & 4.8 & 5.7 & 0.583 \\
CAD & 33.3 & 13.3 & 0.004 \\
Atrial fibrillation history & 23.8 & 8.8 & 0.011 \\
PAD & 11.9 & 7.0 & 0.225 \\
CKD & 35.7 & 7.0 & $<0.001$ \\
Cancer & 16.7 & 8.9 & 0.123 \\
Fever & 95.1 & 93.0 & 0.475 \\
Cough & 42.9 & 51.9 & 0.193 \\
Dyspnea & 81.0 & 66.2 & 0.047 \\
RR & $23.3 \pm 4.9$ & $20.7 \pm 8.0$ & 0.001 \\
HR & $90.3 \pm 18.2$ & $91.3 \pm 16.6$ & 0.221 \\
SO & $91.3 \pm 5.6$ & $93.1 \pm 4.2$ & 0.057 \\
SBP & $132.4 \pm 23.4$ & $132.5 \pm 21.4$ & 0.860 \\
DBP & $80.3 \pm 21.3$ & $75.6 \pm 10.1$ & 0.175 \\
MCA & 28.8 & 32.6 & 0.407 \\
LMWH & 7.5 & 48.7 & $<0.001$ \\
\hline
\end{tabular}

COPD, chronic obstructive pulmonary disease; $\mathrm{CAD}$, coronary artery disease; PAD, peripheral artery disease; CKD, chronic kidney disease; $\mathrm{RR}$, respiratory rate; $\mathrm{HR}$, heart rate; $\mathrm{SO}_{2}$, oxygen saturation; SBP, systolic blood pressure; DBP, diastolic blood pressure; MCA, medical critical area; LMWH, low molecular weight heparin

respiratory rate (RR) $21.3 \pm 7.5$ apm and $\mathrm{SO}_{2} 92.7 \pm 4.6 \%$. Hypertension (56.2\%) was the most prevalent comorbidity. Diabetes, coronary artery disease (CAD), chronic kidney disease (CKD) and history of atrial fibrillation (AF) were present in 18.4, 17.4, 12.9 and $11.9 \%$ of patients respectively; of these latter, only $42 \%$ were already on anticoagulant treatment. Baseline ECG features (Table 2) were characterized by sinus rhythm $(63.7 \%)$ in the vast majority of cases. ST-T abnormalities accounted for $15.9 \%$ of patients, atrial fibrillation for 7.0\%; QTc interval was $449.7 \pm 29.5 \mathrm{~ms}$ at admission. Cardiovascular (CV) comorbidities did not differ in patients with or without ECG alterations. Chronic therapy with antiplatelet therapy or anticoagulant has been found in $22.9 \%$ and $8.9 \%$ of cases. Intensive care unit admission was necessary in $15.9 \%$ of study population; seven patients (3.5\%) underwent cardio-pulmonary resuscitation (Fig. 1). Most of the patients were admitted to internal medicine (IM, 47.3\%), while another consistent part (31.3\%) were treated in medical critical area (MCA, Fig. 2).

30-day overall mortality, provided by electronic medical record, was $20.9 \%$. Compared to survivors, patients who died were older $(79.7 \pm 10.8$ vs $65.6 \pm 14.1, p<0.001)$, with 
Table 2 Baseline ECG characteristics

\begin{tabular}{lllr}
\hline & Dead $(n=42)$ & Alive $(n=159)$ & $p$ \\
\hline Sinus rhythm & 16.4 & 83.6 & $<0.001$ \\
AF & 32.3 & 3.6 & $<0.001$ \\
AVBI & 3.2 & 5.4 & 0.524 \\
RBBB & 16.1 & 9.9 & 0.250 \\
LBBB & 19.4 & 9.0 & 0.102 \\
ST-elevation & 3.3 & 1.8 & 0.518 \\
ST-depression & 20.0 & 4.5 & 0.012 \\
T wave inversion & 3.3 & 15.3 & 0.066 \\
Prolonged QTc & 75.0 & 48.6 & 0.010 \\
QTc & $466.2 \pm 23.6$ & $445.4 \pm 29.4$ & $<0.001$ \\
\hline
\end{tabular}

AF, atrial fibrillation; AVBI, atrio-ventricular block I; RBBB, right bundle branch block; LBBB, left bundle branch block

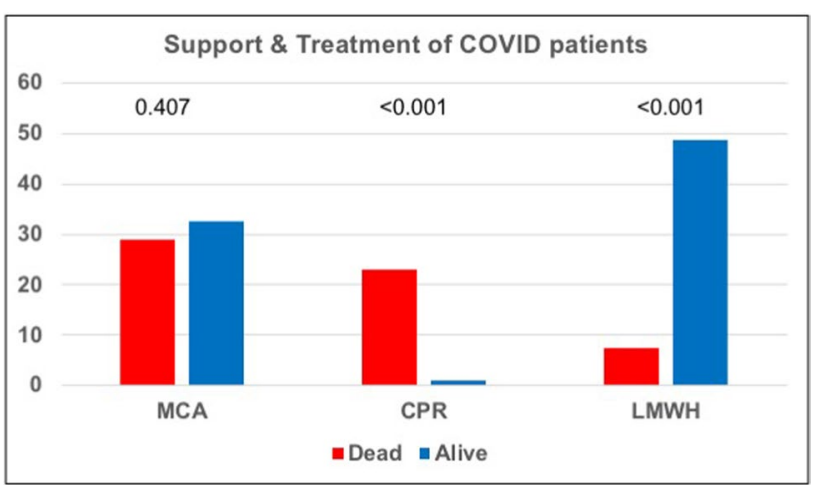

Fig. 1 Support and treatment of COVID19 patients. MCA medical critical area, $C P R$ cardio-pulmonary resuscitation, $L M W H$ lowmolecular weight heparin

\section{COVID-19 patients}

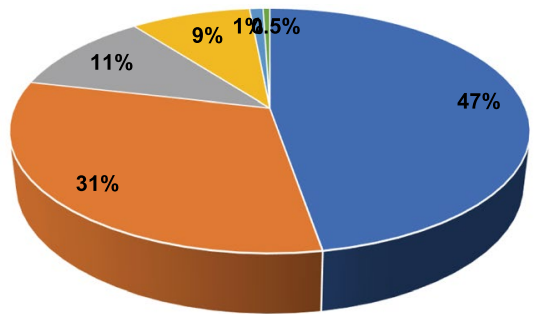

- IM = MCA = Pneumology = ID = Nephrology " ICU

Fig. 2 Destination of COVID-19 patients after admission to the Emergency Department; IM, internal medicine; MCA, medical critical area; ID, infectious diseases; ICU, intensive care unit

a more complex cardiovascular history, including CAD (33.3\% vs $13.3 \%, p=0.004), \mathrm{AF}(23.8$ vs $8.8, p=0.011)$ and CKD (CKD 35.7\% vs 7.0\%, $p<0.001$ ). AF (OR 12.74, 95\%
Table 3 Laboratory parameters at admission

\begin{tabular}{lccr}
\hline & Dead $(n=42)$ & Alive $(n=159)$ & \multicolumn{1}{c}{$p$} \\
\hline TnI & $62.7 \pm 56.4$ & $21.0 \pm 22.3$ & $<0.001$ \\
WBC & $7.8 \pm 3.8$ & $6.8 \pm 3.6$ & 0.102 \\
N/L ratio* & $11.6 \pm 8.9$ & $8.0 \pm 5.2$ & 0.018 \\
CRP & $10.9 \pm 8.0$ & $9.9 \pm 9.9$ & 0.283 \\
LDH & $1089.3 \pm 1821.7$ & $613.9 \pm 328.2$ & 0.010 \\
\hline
\end{tabular}

TnI, troponin I; WBC, white blood cells; N/L, neutrophil to lymphocyte; CRP, C-reactive protein; $\mathrm{LDH}$, lactate dehydrogenase

CI 3.65-44.48, $p<0.001$ ), ST-segment depression (OR 5.30, 95\% CI 1.50-18.81, $p=0.010)$ and QTc-interval prolongation (OR 3.17, 95\% CI 1.24-8.10, $p=0.016$ ) at ECG admission were also associated to an increased mortality risk. On the contrary, sinus rhythm (OR $0.08,95 \%$ CI $0.02-0.27$, $p<0.001$ ) and LMWH administration at admission (OR $0.08,95 \%$ CI $0.02-0.29, p<0.001$ ) were associated with better survival. Laboratory parameters (Table 3 ) did not differ between the two groups, except for baseline $\mathrm{TnI}(62.7 \pm 56.4$ vs $21.0 \pm 22.3, p<0.001)$.

At multivariate analysis, after adjustment for age, sex, diabetes, CAD, resuscitation and MCA admission, sinus rhythm (HR 2.7, CI 95\% 1.1-7.0, $p=0.038$ ) and LMWH (HR 8.5, 95\% CI 2.0-36.6, $p=0.004$ ) were confirmed to be independent predictors of increased survival (Fig. 3).

\section{Discussion}

This is the first study describing clinical and ECG characteristics and 30-day outcome in all-comer patients with a history of COVID-19 pneumonia. In this retrospective realworld cohort of COVID-19 patients we confirmed a high short-term mortality, particularly in patients with a more complex CV history and we obtained the following novel key findings: (1) ECG abnormalities at admission are quite common in COVID-19 infection and related to a higher risk of mortality; (2) sinus rhythm at admission has been associated with a lower mortality even after adjustment for confounding factors such as age, sex, diabetes, CAD, ICU and resuscitation; (3) LMWH has been related, as already shown in other studies [8-11], to lower mortality and furtherly improved survival particularly in patients with sinus rhythm at ECG admission; (4) myocardial biomarkers resulted significantly higher in non-survivors compared to survivors, as expression of a more severe disease with multi-organ failure manifestation.

Poor clinical outcomes in COVID-19 pneumonia patients may be partially explained by underlying severe comorbidities, such as pre-existing CV-conditions [12]. The cytokine storm with hyperinflammation, typical of COVID-19 

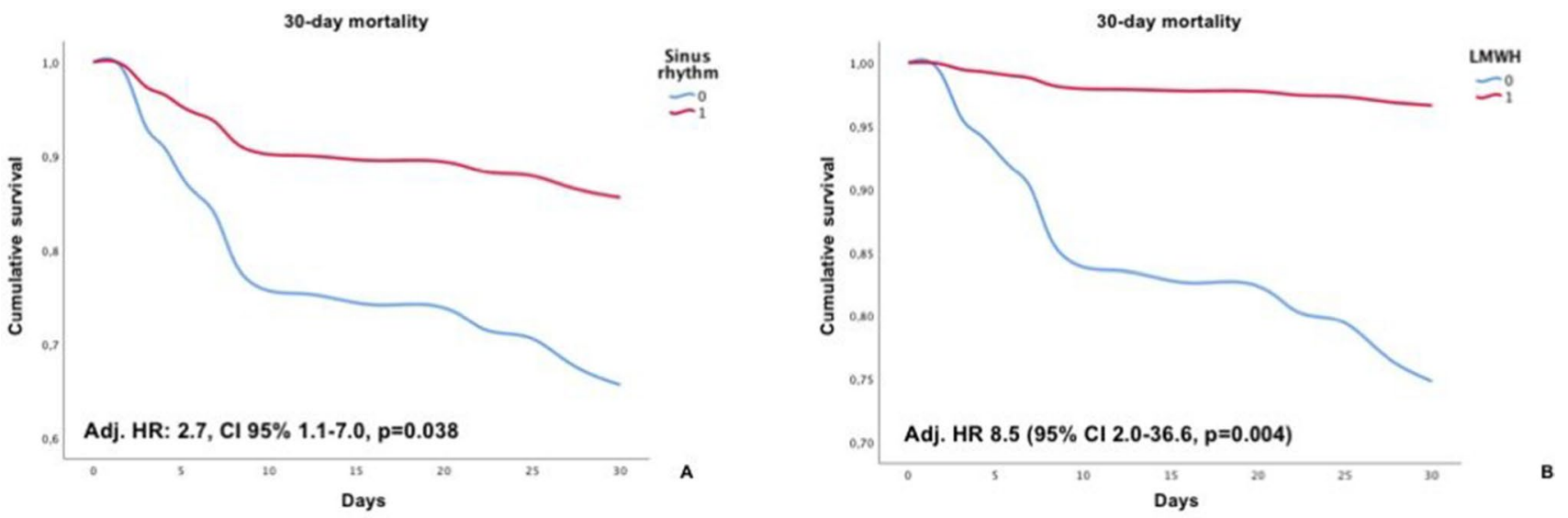

Fig. 3 Cumulative survival of sinus rhythm patients (a) and LWMH administration (b)

infection, leads to multiorgan failure that seems to be the main responsible of high-mortality in this setting [13]. Inflammatory markers are, indeed, elevated in COVID-19 disease and recent acquisitions, suggest a primary role of the endothelium in mediating rheological response between blood compartment and tissues [14].

ECG alterations have been described during hospitalization for COVID-19, reflecting several CV complications ranging from ST-T changes to acute coronary syndrome (ACS), acute peri-myocarditis, pulmonary embolism or rhythm disorders [15-19]. Although these findings confirm that ECG changes during hospitalization may express a wide broad of $\mathrm{CV}$ complications with a potential significant clinical impact on outcome, our results highlighted the importance of ECG abnormalities at admission to stratify mortality risk for COVID-19 patients, deserving a closer monitoring and dedicated therapies.

As of today, arrhythmic manifestation associated with COVID-19 have been only limited described. Atrial fibrillation seems to be the most reported arrhythmia in COVID19 patients, with a prevalence up to $50 \%$ in those needing intensive care [20]. COVID-19 patients affected by more severe infection with ICU admission have been described to develop atrial arrhythmias, as recently reported by University of Alabama at Brimingham [21].

$\mathrm{T}$-wave inversion as well as repolarization abnormalities have been associated to increased risk of mortality in the general population and described in COVID-19 together with myocardial biomarkers increase [22, 23], confirmed also at univariate analysis in our study.

ST-segment elevation, described as an important CV complication of COVID19 infection [24], was rare at admission, but promptly treated with modified diagnostic and treatment algorithm, even a reduction of hospitalization for myocardial infarction has been registered in the COVID-19 era $[25,26]$.
Right bundle branch block (RBBB) has been described in COVID-19 patients as the expected result of an acute right ventricular dysfunction secondary to respiratory failure and associated with worse survival [27].

QT interval prolongation is a dreaded complication of COVID-19 patients and has been described particularly in association with treatment with hydroxychloroquine/ azithromycin [28]. In our cohort, a not negligible portion of patients $(36.8 \%)$ presented a prolonged QTc at admission, underscoring the potential benefit of a greater attention to ECG abnormalities, which may otherwise be overlooked in favor of other less common findings, prior to establishing potential harmful therapeutic regimens. Unfortunately, data regarding drugs administration during hospitalization were not available for our analysis because of the study design, that was focused on patients admission.

Concave ST elevation and PR depression with reciprocal ST depression and PR elevation in avR as expression of acute pericarditis have been also described in COVID-19 patients as a common manifestation [29]. In our analysis, we did not detect, indeed, a significant number of acute pericarditis cases.

In-hospital treatment with anticoagulant therapy has been associated to better prognosis in severe COVID-19 patients, particularly in case of markedly elevated D-dimer [8]. Thrombo-embolic events in COVID-19 patients are not uncommon and probably favored by the exaggerated inflammatory response typical of a considerable portion of COVID-19 infection [30]. Alterations in coagulation parameters, with markedly elevated D-dimer have been associated with worse outcomes and decreased survival in COVID-19 patients [31]. Although anticoagulation was not routinely performed in all patients, it is conceivable that a wider use of anticoagulation at admission, at least at prophylactic dosage, may be related to better outcomes in COVID-19 [8]. 
Increased levels of cardiac biomarkers, particularly troponin, has been described in a considerable portion of COVID-19 patients, as expression of myocardial involvement in multi-organ failure characterized of COVID-19 infection and associated with increased mortality [32]. Troponin seem to be a useful marker for prognosis of COVID19 patients, and has been associated with favorable outcome even in patients with previous CV disease [24].

Mortality in our cohort was higher than reported in China, but in line with data from other regions of Italy [3, 8, 33]. Several conditions (i.e. history of AF, CAD or CKD), laboratory abnormalities (i.e. increased $\mathrm{TnI}$ at admission), instrumental alterations (i.e. ECG abnormalities) and of course older age have been associated with poor outcomes. Older age has been consistently shown to be a major risk factor for poor outcomes [34]. Hypertension and CAD are usually more frequent in non-survivors and hypertension has been related to a higher rate of acute respiratory distress syndrome (ARDS) [35].

The retrospective characteristic of the study and patient selection may have influenced our results, but the strength of our work lies in describing a real-world cohort of allcomer patients admitted to the emergency department for suspected COVID-19 infection, therefore confirmed by routine analyses. Thus, the primary cause of hospitalization had no relation to the $\mathrm{CV}$ background presentation of our patients, not affecting, consequently, ECG characteristics. Our data underline the clinical impact of ECG abnormalities at admission on outcome in COVID-19 patients.

\section{Limitations}

The main limitation of our study is the relatively small size of study population, that limited the statistical power of our analyses. Moreover, given the logistical limitation at the onset of the pandemic infection, some laboratory data and ECG at admission were not collected in all patients. Echocardiography was not routine performed in these patients. The study was designed considering COVID-19 patients at admission; thus data regarding drugs administration or modality of data are lacking. Although data from larger multicenter registries are desirable, are data represent a realworld picture of COVID-19 outbreak in Italy, in one of the most severely affected area as Emilia-Romagna.

\section{Conclusion}

Sinus rhythm at ECG admission in COVID-19 infection was associated with better survival, furtherly improved by LMWH administration, which conferred, moreover, an overall better outcome. Our findings highlight the potential prognostic role of ECG at admission, before respiratory failure manifestations, on outcome. Better prevention of COVID-19 and possibly better evidence-based treatment of COVID-19 is warranted in these patients.

\section{Compliance with ethical standards}

Conflict of interest The authors declare that they have no potential conflict of interest.

Human and animal rights This article does not contain any studies with human participants or animals performed by any of the authors.

Informed consent None.

\section{References}

1. https://www.who.int/emergencies/diseases/novel-coronaviru s-2019/situation-reports-178

2. De Filippo O, D'Ascenzo F, Angelini F et al (2020) Reduced rate of hospital admissions for ACS during Covid-19 outbreak in Northern Italy. N Engl J Med 383(1):88-89. https://doi. org/10.1056/NEJMc2009166

3. Guan WJ, Ni ZY, Hu Y, Liang WH, Ou CQ, He JX, China Medical Treatment Expert Group for COVID-19 et al (2020) Clinical characteristics of coronavirus disease 2019 in China. N Engl J Med. 382:1708-1720

4. Welt FGP, Shah PB, Aronow HD, Bortnick AE, Henry TD, Sherwood MW, American College of Cardiology's Interventional Council and the Society for Cardiovascular Angiography and Interventions et al (2020) Catheterization laboratory considerations during the coronavirus (COVID-19) pandemic: from the ACC's Interventional Council and SCAI. J Am Coll Cardiol. 75:2372-2375

5. Li Y, Liu T, Tse G, Wu M, Jiang J, Liu M, Tao L (2020) Electrocardiograhic characteristics in patients with coronavirus infection: a single-center observational study. Ann Noninvasive Electrocardiol 20:e12805

6. Bertini M, Ferrari R, Guardigli G, Malagù M, Vitali F, Zucchetti O, D’Aniello E, Volta CA, Cimaglia P, Piovaccari G, Corzani A, Galvani M, Ortolani P, Rubboli A, Tortorici G, Casella G, Sassone B, Navazio A, Rossi L, Aschieri D, Rapezzi C (2020) Electrocardiographic features of 431 consecutive, critically ill COVID-19 patients: an insight into the mechanisms of cardiac involvement. Europace. 18:euaa258

7. Angeli F, Spanevello A, De Ponti R, Visca D, Marazzato J, Palmiotto $\mathrm{G}$ et al (2020) Electrocardiographic features of patients with COVID-19 pneumonia. Eur J Intern Med. https://doi. org/10.1016/j.ejim.2020.06.015

8. Tang N, Bai H, Chen X, Gong J, Li D, Sun Z (2020) Anticoagulant treatment is associated with decreased mortality in severe coronavirus disease 2019 patients with coagulopathy. J Thromb Haemost 18(5):1094-1099

9. Mattioli M, Benfaremo D, Mancini M, Mucci L, Mainquà $\mathrm{P}$, Polenta A et al (2020) Safety of intermediate dose of low molecular weight heparin in COVID-19 patients. J Thromb Thrombolysis 13:1-7

10. Paolisso P, Bergamaschi L, D’Angelo EC, Donati F, Giannella M, Tedeschi S et al (2020) Preliminary experience with low 
molecular weight heparin strategy in COVID-19 patients. Front Pharmacol 6(11):1124

11. Desai A, Voza G, Paiardi S, Teofilo FI, Caltagirone G, Pons MR, Humanitas COVID-19 task force et al (2020) The role of anti-hypertensive treatment, comorbidities and early introduction of LMWH in the setting of COVID-19: A retrospective, observational study in Northern Italy. Int J Cardiol. https://doi. org/10.1016/j.ijcard.2020.09.062

12. Inciardi RM, Adamo M, Lupi L, Cani DS, Di Pasquale M, Tomasoni D, Italia L, Zaccone G, Tedino C, Fabbricatore D, Curnis A, Faggiano P, Gorga E, Lombardi CM, Milesi G, Vizzardi E, Volpini M, Nodari S, Specchia C, Maroldi R, Bezzi M, Metra M (2020) Characteristics and outcomes of patients hospitalized for COVID-19 and cardiac disease in Northern Italy. Eur Heart J 41(19):1821-1829

13. Mehta P, McAuley DF, Brown M, Sanchez E, Tattersall RS, Manson JJ, Healh Across Speciality Collaboration UK (2020) COVID19: consider cytokine storm syndromes and immunosuppression. Lancet 395:1033-1034

14. Libby P, Lüscher T (2020) COVID-19 is, in the end, an endothelial disease. Eur Heart J 41(32):3038-3044

15. Mountantonakis SE, Saleh M, Coleman K, Kuvin J, Singh V, Jauhar R et al (2020) Out-of-hospital cardiac arrest and acute coronary syndrome hospitalizations during the COVID-19 surge. J Am Coll Cardiol S0735-1097(20):35964-35967

16. Doyen D, Moceri P, Ducreux D, Dellamonica J (2020) Myocarditis in a patient with COVID-19: a cause of raised troponin and ECG changes. Lancet 395(10235): 1516

17. Clerkin KJ, Fried JA, Raikhelkar J, Sayer G, Griffin JM, Masoumi A et al (2020) Coronarvirus disease 2019 (COVID-19) and cardiovascular disease. Circulation 141(20):1648-1655

18. Casey K, Iteen A, Nicolini R, Auten J (2020) COVID-19 pneumonia with hemoptysis: acute segmental pulmonary emboli associated with novel coronavirus infection. Am J Emerg Med 38(7):1544.e1-1544.e3

19. Kochav SM, Coromilas E, Nalbandian A, Ranard LS, Gupta A, Chung MK et al (2020) Cardiac arrhythmias in COVID-19 infection. Circ Arrhythm Electrophysiol 13(6):e008719

20. Wang D, Hu B, Hu C, Zhu F, Liu X, Zhang J et al (2020) Clinical Characteristics of 138 hospitalized patients With 2019 novel coronavirus-infected pneumonia in Wuhan, China. JAMA 323(11):1061-1069

21. Bhatla A, Mayer MM, Adusumalli S, Hyman MC, Oh E, Tierney A et al (2020) COVID-19 and cardiac arrhythmias. Heart Rhythm S1547-5271(20):30594-30604

22. Daviglus ML, Liao Y, Greenland P, Dyer AR, Liu K, Xie X (1999) Association of nonspecific minor ST-T abnormalities with cardiovascular mortality: the Chicago Western Electric Study. JAMA 282:530-536

23. Guo T, Fan Y, Chen M, Wu X, Zhang L, He T (2020) Cardiovascular implications of fatal outcomes of patients with coronavirus disease 2019 (COVID-19). JAMA Cardiol. 5(7):1-8
24. Bangalore S, Sharma A, Slotwiner A, Yatskar L, Harari R, Shah B (2020) ST-segment elevation in patients with covid-19-a case series. N Engl J Med 382:2478-2480

25. Chieffo A, Stefanini GG, Price S, Barbato E, Tarantini G, Karam $\mathrm{N}$ et al (2020) EAPCI position statement on invasive management of acute coronary syndromes during the COVID-19 pandemic. Eur Heart J. 41(19):1839-1851

26. De Rosa S, Spaccarotella C, Basso C, Calabrò MP, Curcio A, Filardi PP et al (2020) Reduction of hospitalizations for myocardial infarction in Italy in the COVID-19 era. Società Italiana di Cardiologia and the CCU Academy investigators group. Eur Heart J. 41(22):2083-2088

27. McCullough SA, Goyal P, Krishnan U, Choi JJ, Safford MM, Okin PM (2020) Electrocardiographic findings in coronavirus disease-19: insights on mortality and underlying myocardial processes. Card Fail 26(7):626-632

28. Chorin E, Wadhwani L, Magnani S, Dai M, Shulman E, Nadeau-Routhier C et al (2020) QT interval prolongation and torsade de pointes in patients with COVID-19 treated with hydroxychloroquine/azithromycin. Heart Rhythm S1547-5271(20):30435-30445

29. Fried JA, Ramasubbu K, Bhatt R, Topkara VK, Clerkin KJ, Horn $E$ et al (2020) The variety of cardiovascular presentations of COVID-19. Circulation 141(23):1930-1936

30. Glynn RJ, Ridker PM (2018) Inflammation, venous thromboembolism, and what we can do about it. Eur Heart J 39:3615-3617

31. Tang N, Li D, Wang X, Sun Z (2020) Abnormal coagulation parameters are associated with poor prognosis in patients with novel coronavirus pneumonia. J Thromb Haemost 18:844-847

32. Tresalvi G, Vincenzi M, Calabretta D, Biasco L, Pedrazzini G, Winterton D (2020) Elevated troponin in patients with coronavirus disease 2019: possible mechanisms. Card Fail 26(6):470-475

33. Chen N, Zhou M, Dong X, Qu J, Gong F et al (2020) Epidemiological and clinical characteristics of 99 cases of 2019 novel coronavirus pneumonia in Wuhan, China: a descriptive study. Lancet 395:507-513

34. Zhou F, Yu T, Du R, Fan G, Liu Y, Liu Z et al (2020) Clinical course and risk factors for mortality of adult inpatients with COVID-19 in Wuhan, China: a retrospective cohort study. Lancet 395:1054-1062

35. Wu C, Chen X, Cai Y, Xia J, Zhou X, Xu S et al (2020) Risk factors associated with acute respiratory distress syndrome and death in patients with coronavirus disease 2019 pneumonia in Wuhan, China. JAMA Intern Med. https://doi.org/10.1001/jamai nternmed.2020.0994

Publisher's Note Springer Nature remains neutral with regard to jurisdictional claims in published maps and institutional affiliations. 